\title{
Why current peptide-based cancer vaccines fail: lessons from the three Es
}

“Equillibrium results in a stationary, clinically undetectable 'dormant' tumor for periods of months to years, eventually for a lifetime, an interval in which the adaptive immune system sculptures or edits the tumor cells, thereby (unfortunately) rendering them less and less immunogenic."

According to recently published reviews on the efficacy of cancer vaccines, including 20 uncontrolled therapeutic peptide-based vaccine trials in melanoma patients [1] and ten trials in colorectal cancer (CRC) patients with advanced disease [2], only eight of the 327 melanoma patients showed a complete, probably vaccine-induced, response and only one of 118 patients with advanced CRC displayed a partial response. It is thus generally realized that the clinical efficacy of therapeutic cancer vaccines is poor. An overall objective response rate of $3.3 \%$ was observed in 1306 vaccine treatments (including some of those trials mentioned above) of cancer patients with advanced disease [3]. However, a few trials have reported objective response rates as high as $24 \%$ $[4,5]$. In these uncontrolled studies, patients were vaccinated with pools of known tumor-associated HLA class I-binding peptides for which the individual patient showed T-cell reactivity, and in one of the trials vaccination was combined with low-dose alkylating chemotherapy [5].

\section{'...a dynamic model for immunosurveillance including both the individual tumor's biology and patient immunity: the three Es of cancer immunoediting ... in which the three Es stand for tumor elimination, equilibrium and escape.'}

In most studies, discoveries of immunogenic tumor-associated HLA class I-restricted antigenic peptides (TAAp) for vaccination have been based on the use of tumor-reactive $T$ cells derived from peripheral blood of cancer patients with advanced disease. Initially, a direct genetic approach using a tumor cDNA-expression cloning technique was employed [6]; later on, socalled reverse immunology took over as the stateof-the-art technology to detect and characterize new TAAp [7-9]. To date, hundreds of TAAp, for

which $\mathrm{T}$ cells of cancer patients and sometimes also healthy individuals have shown reactivity, have been tested in clinical trials. However, not a single TAAp-based vaccine has gained official drug approval owing to lack of proven clinical efficacy. Lack of approval is not due to technical problems or toxicity since highly purified peptides with known HLA class I-binding affinities can be easily synthesized at GMP levels and appear to be without severe side effects such as local toxicity or autoimmunity.

\section{"It is thus generally realized that the clinical efficacy of therapeutic cancer vaccines is poor. An overall objective response rate of $3.3 \%$ was observed in 1306 vaccine treatments.'}

The majority of strategies for antigen/epitope discovery are based on the classical concept of tumor immunity and immunosurveillance [10]. TAAp used for vaccination are defined by their capability to elicit tumor-associated reactivity of $\mathrm{T}$ cells in cancer patients. Schreiber $e t$ al. have during the years worked out a dynamic model for immunosurveillance including both the individual tumor's biology and patient immunity: the three Es of cancer immunoediting $[11,12]$ in which the three Es stand for tumor elimination, equilibrium and escape. If this hypothesis is correct, it may partly explain the lack of clinical results obtained in TAAp-based vaccination trials so far and suggest new rationales for the development more efficient cancer epitope-finding strategies.

According to the three Es hypothesis, which is supported by extensive mouse experimental work [11] and by a magnitude of random clinical observations on tumor dormancy in apparently healthy individuals (reviewed in [13]), newly arising tumor cells may be rejected soon after their formation by both the innate and the adaptive

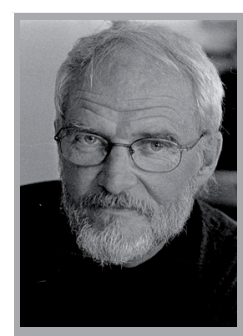

Mogens H Claesson

Department of International

Health, Immunology \&

Microbiology, The Faculty of

Health Sciences, University of

Copenhagen, Copenhagen,

Denmark

Tel.: +4535327270

Fax: +4535327269

claesson@sund.ku.dk

\section{future}


immune system. This is the elimination stage of the three Es hypothesis and is also the core of classic immunosurveillance according to which neoplastic cells are recognized and rejected due to somatic mutations making the cells visible targets for innate and in particular adaptive immunity [10]. If elimination is unsuccessful, tumors, according to the three Es hypothesis, develop into a state of equilibrium, in which their potential expansion is constrained by adaptive immunity. Equillibrium results in a stationary, clinically undetectable 'dormant' tumor for periods of months to years, eventually for a lifetime, an interval in which the adaptive immune system sculptures or edits the tumor cells, thereby (unfortunately) rendering them less and less immunogenic. When fully edited, the tumor cells have lost or downregulated most of their antigenicity that makes them prone to escape the immunological constrains, and enter into the stage of escape in which they become clinically observable.

\section{'Although TAAp derived from melanoma antigens in general perform poorly in vaccines, in adoptive TIL transfers, the number of tumor antigen-specific T-effector cells at the tumor site is far above that obtained by vaccination, being high enough to react with and kill tumor cells in the escape stage.'}

The demonstration of immune reactivity against TAAp in the individual cancer patient, may, in most cases, as implicated by the three Es hypothesis, reflect immunological memory for tumor antigenicity expressed during the period of immunosculpturing in the stage of tumor-host equilibrium. Later on, in the escape stage, when the tumor, driven by the immune system has downmodulated its antigenicity, the tumor cells are less sensitive to the previously generated antitumor immunity. Isolated clones of TAAp reactive peripheral blood T cells the discovery base for new TAAp - may well show killing of in vitro-expanded autologous tumor cell lines because of the artificially high effector-to-tumor cell ratio in the in vitro cytotoxicity experiments and/or because tumor cell lines, in the absence of ongoing immunoediting during culture in vitro, may regain some of their former antigenicity. However, as documented in the many clinical trials, although TAAp vaccination in many cases leads to excellent vaccine recognition by $\mathrm{T}$ cells [14], there appears, in the majority of cases, to be a general lack of correlation between T-cellmediated tumor reactivity in vitro and in vivo, although such correlations have been documented in a few vaccine trials using mixtures of TAAp for which the patient, prior to vaccination, shows immune reactivity [14].

'Thus TILs, in particular in responding patients, may be the most relevant T-cell reagents in the screening for new TAAp.'

As the three Es hypothesis predicts, those TAAp identified by the reactivity of peripheral blood T cells of cancer patients are in most cases deemed to be ineffective as a therapeutic vaccine. T-cell reactivity in the patient reflects immunological memory against tumor antigens lost in the process of immunoediting and these antigens are therefore, in most cases, not vaccine relevant. In some responding patients, vaccinespecific $T$ cells have been shown to lead to generation of new clonotypes of tumor-infiltrating cytotoxic T cells (TILs) with new specificities, however, not driven by the antigenicity of the vaccine [15-17]. These TILs recognize TAAp still presented by tumor cells in the escape stage and they are, therefore, capable of killing the tumor. Thus TILs, in particular in responding patients, may be the most relevant T-cell reagents in the screening for new TAAp as demonstrated initially by the Rosenberg group who by the use of TILs discovered a number of melanocyte differentiation antigens upregulated in melanomas $[18,19]$. Similarly, the peripheral blood of patients cured by immunotherapy might be another good source of T cells with specificity for TAA expressed during the tumor-escape phase. In line with this latter possibility, we are currently examining reactivity against overlapping TAAp in $T$ cells from peripheral blood of patients cured from advanced renal cancer by IL-2 treatment [20]. In support of the use of TILs or T lymphocytes from patients cured by immunotherapy, such cells (TILs) have shown remarkable antitumor activity after in vitro expansion and adoptive transfer to cancer patients $[21,22]$. Some TIL preparations with high clinical efficacy recognize melanoma antigens against which reactivity is readily detectable in many patients. Although TAAp derived from melanoma antigens in general perform poorly in vaccines [1], in adoptive TIL transfers, the number of tumor antigen-specific T-effector cells at the tumor site is far above that obtained by vaccination [16], being high enough to react with and kill tumor cells in the escape stage. 
With the use of TILs or peripheral blood $T$ cells from the few patients who benefit from immunotherapy, TAAp screening will hopefully disclose new classes of immunogenic TAA of use in tumor vaccines. While the advantage is that TAA discovered in recovering patients may represent real and strong tumor-regression antigens, these TAAp may only be capable of raising antitumor immunity in patients already responding to immunotherapy. This pessimistic view is, as mentioned above, supported by the general lack of clinical efficacy even in vaccines containing one or more TAAp [1], initially discovered by the use of TILs in the direct genetic approach $[18,19]$.

\section{'In some responding patients, vaccine- specific $T$ cells have been shown to lead to generation of new clonotypes of tumor- infiltrating cytotoxic T cells (TILs) with new specificities, however, not driven by the antigenicity of the vaccine.'}

To increase the chance for responsiveness and to limit the risk that vaccine-induced immunoediting and TAA downmodulation a polyepitopic vaccine approach using a diversity of proven tumor regression peptides should always be considered [23].

Obviously, downmodulation of TAA is not the only explanation for the failure of TAApbased therapeutic cancer vaccination, and the immune editing hypothesis is a simplistic animal model not fully reflecting the much more complex phenomenology of tumor immunity in humans. In addition to downmodulation of TAA during the process of immunoedition, other constrains to successful vaccination are local and systemic suppression mediated by the growing tumor and its environment including activation and stimulation of Tregs, tolerogenic dendritic cells, myeloid suppressor cells and neoangiogenic factors, all elements that will counteract active as well as passive immunotherapy [24].

In conclusion, a successful therapeutic cancer vaccine should always be polytopic and include a variety of tumor-associated peptides expressed by the patient's own growing tumor. In addition, measures should be taken to reduce the immunity hostile tumor environment, including cells and factors that suppress immunity, and to stimulate vaccine-induced lymphocyte proliferation in the patients.

\section{Financial \& competing interests disclosure}

The author has no relevant affiliations or financial involvement with any organization or entity with a financial interest in or financial conflict with the subject matter or materials discussed in the manuscript. This includes employment, consultancies, honoraria, stock ownership or options, expert testimony, grants or patents received or pending, or royalties.

No writing assistance was utilized in the production of this manuscript.

\section{Bibliography}

1 Engell-Noerregaard L, Hansen TH, Andersen MH, Thor Straten P,

Svane IM: Review of clinical studies on dendritic cell-based vaccination of patients with malignant melanoma: assessment of correlation between clinical response and vaccine parameters. Cancer Immunol. Immunother. 58, 1-14 (2009).

2 Nagorsen D, Thiel E: Clinical and immunologic responses to active specific cancer vaccines in human colorectal cancer. Clin. Cancer Res. 12, 3064-3069 (2006).

3 Lowy DR, Schiller JT: Preventive cancer vaccines. In: DeVita, Hellman and Rosenberg's Cancer: Principles \& Practice of Oncology. Vincent T, DeVita VT Jr, Hellman S, Rosenberg SA (Eds). Lippincott Williams \& Wilkins, Philadelphia, PA, USA, 351-368 (2008).
4 Yajima N, Yamanaka R, Mine T et al: : Immunologic evaluation of personalized peptide vaccination for patients with advanced malignant glioma. Clin. Cancer Res. 11, 5900-5911 (2005).

5 Noguchi M, Mine T, Yamada A et al.: Combination therapy of personalized peptide vaccination and low-dose estramustine phosphate for metastatic hormone refractory prostate cancer patients: an analysis of prognostic factors in the treatment. Oncol. Res. 16, 341-349 (2007).

6 van der Bruggen $P$, Traversari C, Chomez $\mathrm{P}$ et al:: A gene encoding an antigen recognized by cytolytic $\mathrm{T}$ lymphocytes on a human melanoma. Science 254, 1643-1647 (1991).

7 Boon T, van der Bruggen P: Human tumor antigens recognized by $\mathrm{T}$ lymphocytes.

J. Exp. Med. 183, 725-729 (1996).

8 Maecker B, von Bergwelt-Baildon, Anderson KS, Vonderheide RH, Schultze JL:
Linking genomics to immunotherapy by reverse immunology - 'immunomics' in the new millennium. Curr. Mol. Med. 1, 609-619 (2001).

9 Tanzarella S, Fleischhauer K, van Endert P, Bordignon C, Traversari C: Characterization of antigenic peptide epitopes by reverse immunology: induction of cytotoxic $\mathrm{T}$ lymphocytes specific for exogenous peptide only. Int. J. Cancer 72 , 912-915 (1997)

10 Burnet FM: Cancer - a biological approach. Brit. Med. J. 1, 841-847 (1957).

11 Schreiber RD: Adaptive immunity maintains occult cancer in an equilibrium state. Nature 450, 903-907 (2007).

12 Teng MW, Swann JB, Koebel CM, Schreiber RD, Smyth MJ: Immunemediated dormancy: an equilibrium with cancer. J. Leukoc. Biol. 84, 988-993 (2008). 
13 Lars A, Naumov GN, Folkman J: Tumor dormancy. Acta Pathologica, Microbiologica et Immunologica Scandinavica 116 (2008).

14 Itoh K, Yamada A, Mine T, Noguchi M: Recent advances in cancer vaccines: an overview. Jpn J. Clin. Oncol. 39, 73-80 (2009).

15 Germeau C, Ma W, Schiavetti F et al.: High frequency of antitumor $\mathrm{T}$ cells in the blood of melanoma patients before and after vaccination with tumor antigens. J. Exp. Med. 201, 241-248 (2005).

16 Théate I, van Baren N, Coulie PG, Boon T: Contrasting frequencies of anti-tumor and anti-vaccine $T$ cells in metastases of a melanoma patient vaccinated with a MAGE tumor antigen. J. Exp. Med. 201, 249-257 (2005).
17 Carrasco J, Van Pel A, Neyns B et al.: Vaccination of a melanoma patient with mature dendritic cells pulsed with MAGE-3 peptides triggers the activity of nonvaccine anti-tumor cells. J. Immunol. 180, 3585-3593 (2008).

18 Rosenberg SA, Yang JC, White DE, Steinberg SM: Durability of complete responses in patients with metastatic cancer treated with high-dose interleukin-2: identification of the antigens mediating response. Ann. Surg. 228, 307-319 (1998).

19 Rosenberg SA: Cancer vaccines based on the identification of genes encoding cancer regression antigens. Immunol. Today 18 , 175-182 (1997).

20 Donskov F, Middleton M, Fode K et al.: Two randomised Phase II trials of subcutaneous interleukin-2 and histamine dihydrochloride in patients with metastatic renal cell carcinoma. Br. J. Cancer 93 , 757-762 (2005).
21 Dudley ME, Yang JC, Sherry R et al.: Adoptive cell therapy for patients with metastatic melanoma: evaluation of intensive myelo-ablative chemoradiation preparative regimens. J. Clin. Oncol. 26, 5233-5239 (2008).

22 Rosenberg SA, Dudley ME: Adoptive cell therapy for the treatment of patients with metastatic melanoma. Curr. Opin. Immunol. 21(2), 233-240 (2009).

23 Buus S, Claesson MH: Identifying multiple tumor-specific epitopes from large-scale screening for overexpressed mRNA. Curr. Opin. Immunol. 16, 137-142 (2004).

24 Gilboa E: DC-based cancer vaccines. J. Clin. Invest. 117, 1195-1203 (2007). 